\title{
La construction des politiques pharmaceutiques nationales à l'épreuve des programmes de santé globale
}

L'approvisionnement des médicaments contre le paludisme au Bénin et au Ghana

Jessica Pourraz, Carine Baxerres et Maurice Cassier

\section{OpenEdition}

\section{Journals}

Édition électronique

URL : http://journals.openedition.org/anthropodev/706

DOI : 10.4000/anthropodev.706

ISSN : 2553-1719

Éditeur

APAD - Association pour l'anthropologie du changement social et du développement

Édition imprimée

Date de publication : 1 décembre 2018

Pagination : 169-192

ISBN : 979-10-93476-06-3

ISSN : 2276-2019

Référence électronique

Jessica Pourraz, Carine Baxerres et Maurice Cassier, « La construction des politiques

pharmaceutiques nationales à l'épreuve des programmes de santé globale », Anthropologie \&

développement [En ligne], 48-49 | 2018, mis en ligne le 18 juillet 2019, consulté le 19 août 2019. URL

http://journals.openedition.org/anthropodev/706 ; DOI : 10.4000/anthropodev.706

La revue Anthropologie \& développement est mise à disposition selon les termes de la Licence Creative Commons Attribution 4.0 International. 


\section{La construction des politiques pharmaceutiques nationales à l'épreuve des programmes de santé globale}

L'approvisionnement des médicaments contre le paludisme au Bénin et au Ghana

Jessica Pourraz, Carine Baxerres et Maurice Cassier

Le Ghana et le Bénin dépendent des subventions internationales pour approvisionner en médicaments antipaludiques leurs secteurs publics - et pour une partie le secteur privé ghanéen à partir de 2010. Les acteurs transnationaux construisent des réseaux d'approvisionnement qui entrent en concurrence avec les dispositifs nationaux existants. La multiplication de ces circuits engendre une fragmentation du système d'approvisionnement qui conduit à des dysfonctionnements dans la gestion des médicaments au niveau national. Les acteurs transnationaux cherchent alors à impulser certaines formes d'appropriation auprès des États afin de répondre aux problèmes d'inefficacité des systèmes d'approvisionnement qu'ils ont générés. Le Ghana, disposant d'un important tissu industriel, tente de développer une production locale d'antipaludiques, alors que le Bénin envisage des moyens pour reprendre le contrôle sur les importations. Cet article interroge le concept d'appropriation des politiques de développement par le prisme des politiques de mise à disposition des antipaludiques au Bénin et au Ghana. En analysant des dispositifs institutionnels nationaux mis en œuvre par les pays avec le soutien des acteurs transnationaux, cette contribution éclaire les relations de pouvoir et le degré de contrôle qu'exercent les institutions nationales sur la définition et la mise en œuvre de leur politique, qui restent largement dominées par les acteurs transnationaux.

Ghana and Benin are dependent on international grants to supply their public sectors with antimalarial medicines and for part of Ghana's private sector since 2010. Transnational actors generate new supply networks that compete with existing national supply systems. The multiplication of these circuits generates a 
fragmentation of the supply system that leads to dysfunctions in the management of medicines at the national level. Transnational actors then seek to stimulate ownership in both countries in order to address inefficiencies in the supply systems they have generated. Ghana, with a large industrial production capacity, is trying to develop local antimalarial drugs production, while Benin is considering ways to regain control over imports. This article examines the concept of ownership of development policies through the prism of antimalarial drugs policies in Benin and Ghana. By analyzing institutional mechanisms implemented by countries with the support of transnational actors, this contribution sheds light on the power relations and the degree of control exercised by national institutions in the definition and implementation of their policies, which remain largely dominated by transnational actors.

\section{Introduction}

Suite à l'apparition en Afrique à la fin des années 1980 de résistances aux traitements utilisés contre le paludisme, l'Organisation mondiale de la santé (OMS) recommande à partir de 2006 l'usage des Combinaisons thérapeutiques à base d'artémisinine (CTA). Les CTA étant bien plus onéreuses que les anciens traitements, leur financement devient un enjeu majeur pour les pays. L'arrivée sur le continent africain des CTA s'inscrit dans un moment particulier de reconfiguration politique et économique des programmes de santé internationale. La notion de santé globale, devenue omniprésente au cours des 20 dernières années, remplace progressivement les concepts de santé internationale et de médecine tropicale. Les programmes de santé globale constituent une arène d' " acteurs transnationaux " (Eboko et al., 2011), composée de fondations privées, de partenariats public-privé, de programmes bilatéraux et d'ONG. Ils participent d'une nouvelle gouvernance de la santé qui se définit par " the formal and informal institutions, norms and processes which govern or directly influence global health policy and outcomes" (Sridhar, 2009: 1366).

L'arrivée des CTA en Afrique coïncide aussi avec l'émergence d'un nouveau paradigme de l'aide publique au développement. En réponse aux difficultés engendrées par les politiques d'ajustement structurel, les 
institutions financières de Bretton Woods affichent une volonté de rompre avec leurs pratiques antérieures et promeuvent l'appropriation des politiques de développement par les pays (Raffinot, 2010). Le changement de paradigme est officialisé en 2005 lors de la signature de la déclaration de Paris par les pays, les institutions financières et les organisations internationales. Elle vise à pallier l'inefficacité et la fragmentation des programmes de santé globale, en encourageant l'appropriation des politiques de développement par les pays qu'elle définit comme l'exercice par les États d'un leadership et d'une réelle maîtrise sur les politiques, les stratégies de développement et la coordination des acteurs (Sridhar, 2009). Pour ce faire, elle encourage l'harmonisation, l'alignement et la cohérence entre les politiques nationales et celles des acteurs transnationaux. Mais ces engagements peinent à se concrétiser dans le domaine de la santé ce qui s'explique par des facteurs structurels tels que l'importance des moyens financiers des acteurs transnationaux bien supérieurs à ceux des États, ainsi que la forte augmentation du nombre et du type d'acteurs impliqués qui caractérisent la gouvernance de la santé globale (Sridhar, 2009).

Le Bénin et le Ghana possèdent des systèmes pharmaceutiques différents hérités de la période coloniale et des politiques de développement adoptées à leur indépendance. Si le Ghana, qui compte aujourd'hui 36 firmes pharmaceutiques, a développé une production locale de médicaments dès 1957 avec le recours à des multinationales étrangères, le Bénin a longtemps privilégié la sauvegarde du système d'approvisionnement hérité de l'époque coloniale basé sur les importations de médicaments via la France principalement (Baxerres, 2013). Les industries locales au Ghana approvisionnent à hauteur d'un tiers de ses besoins le secteur privé, le reste des médicaments étant importés d'Asie (Chaudhuri, 2015). Le secteur pharmaceutique privé au Ghana est plus important qu'au Bénin avec plus de 600 grossistes privés et un grossiste public, le Central Medical Store; celui-ci joue un rôle bien moins important que la Centrale d'achat des médicaments essentiels et consommables médicaux (CAME) du Bénin pour l'approvisionnement des structures sanitaires publiques (Mahamé et Baxerres, 2015). Les deux pays sont inégalement équipés en matière d'appareils réglementaires. Le Bénin dispose d'une Direction des pharmacies, du médicament et des 
explorations diagnostiques (DPMED) et le Ghana de la Food and Drugs Authority (FDA Ghana) dont l'autonomie et les ressources humaines et financières sont bien supérieures ${ }^{1}$.

Malgré ces singularités, les deux pays dépendent entièrement des subventions internationales pour l'achat des CTA distribuées dans leur secteur public - et, à partir de 2010, pour une partie dans le secteur privé ghanéen. Les aides financières sont conditionnées à l'achat de médicaments préqualifiés par l'OMS ${ }^{2}$, certification dont les firmes ghanéennes ne bénéficient pas ce qui les exclut de ce marché. Deux principales initiatives de santé globale financent l'achat des CTA dans les deux pays. II s'agit du Fonds mondial de lutte contre le sida, la tuberculose et le paludisme, institution multilatérale créée en 2001 par le secrétaire général des Nations unies Kofi Annan, et qui est surtout prépondérante au Ghana; et du programme bilatéral américain, Presidential's Malaria Initiative (PMI), créé en 2005 par le président Georges W. Bush, plus présente au Bénin. Dans ces conditions de dépendance (Naudet, 2000), de quelle autonomie disposent les États pour la construction de leurs politiques pharmaceutiques?

Cet article interroge le concept d'appropriation des politiques de mise à disposition des CTA par le Bénin et le Ghana en analysant les dispositifs institutionnels nationaux mis en œuvre par chacun de ces pays avec le soutien des acteurs transnationaux. Le concept d'appropriation défini comme " la maîtrise des politiques " (Raffinot, 2010 : 89) est utile ici pour analyser le degré de contrôle qu'exercent les institutions nationales sur les choix des dispositifs et leur mise en œuvre. II permet d'interroger plus globalement la question des rapports de pouvoir entre acteurs nationaux et transnationaux dans la construction des politiques pharmaceutiques nationales. Ce concept, bien qu'emprunté aux

1 Ainsi la DPMED emploie six pharmaciens alors que la FDA-Ghana en compte une cinquantaine.

2 Un médicament générique est la copie d'un médicament dont le brevet a expiré. La préqualification de l'OMS garantit la qualité et la similarité du médicament générique par rapport au médicament de référence. Elle exige que les usines soient conformes aux normes des Bonnes Pratiques de Fabrication (BPF) et que le générique soit bioéquivalent au médicament qu'il copie (Lantenois et Coriat, 2014). 
professionnels du développement, nous semble pertinent car il permet d'interroger, dans le sillage d'une littérature critique de l'influence des programmes de santé globale sur les processus de décision et de fabrication des politiques publiques nationales, les défis et les limites de l'appropriation des politiques de développement par les États (Esser, 2014 ; Raffinot, 2010 ; Sridhar, 2009). Toutefois, nous proposons à travers cet article, d'enrichir l'analyse de la fabrique des politiques publiques par la prise en compte des acteurs économiques que sont les firmes pharmaceutiques et de l'histoire des politiques de développement industriel des pays. Les apports historiques, tout comme le rôle des acteurs non étatiques, sont souvent écartés des travaux interrogeant l'influence des acteurs transnationaux (Gautier et Ridde, 2017). Notre contribution s'inspire également des travaux en histoire et en sociologie des sciences (Cassier et Correa, 2009) qui permettent d'interroger le rôle et le pouvoir des États du Bénin et du Ghana par le prisme des techniques et des savoirs appliqués aux politiques pharmaceutiques. Ceci permet de renforcer les analyses des politiques publiques que proposent les sciences politiques et l'anthropologie du développement. L'originalité de cette contribution repose sur la comparaison de deux pays ayant adopté des politiques différentes en matière de développement, mais qui demeurent confrontés aux mêmes enjeux dans l'appropriation de leurs politiques pour la mise à disposition des CTA.

\section{Contexte empirique de l'étude et méthodologie}

Notre analyse s'appuie sur un recueil de données ${ }^{3}$ réalisé durant 14 mois entre 2014 et 2017 au Bénin et au Ghana ${ }^{4}$. Elle se fonde sur des entretiens

${ }^{3}$ Ce travail est tiré d'une thèse de sociologie réalisée par Jessica Pourraz, et encadrée par Maurice Cassier qui apporte ainsi sa contribution en histoire et sociologie des sciences et par Carine Baxerres qui contribue par sa connaissance des systèmes pharmaceutiques du Bénin et du Ghana. Cette thèse a été réalisée dans le cadre du programme de recherche pluridisciplinaire dirigé par Carine Baxerres : " Globalmed - les combinaisons thérapeutiques à base d'artémisinine (CTA) : une illustration du marché global du médicament, de l'Asie à l'Afrique ", financé par l'European Research Council pour une durée de cinq ans (2014-2019) dans le cadre du $7^{\mathrm{e}}$ programme cadre de l'Union européenne (FP7/2007-2013) / ERC grant 
semi-directifs $(n=97)$ conduits avec les acteurs transnationaux impliqués dans l'achat des CTA, les acteurs nationaux en charge des politiques de lutte contre le paludisme, les acteurs transnationaux soutenant la production locale au Ghana et les firmes pharmaceutiques ghanéennes. Les thématiques abordées lors des entretiens concernaient les relations entre acteurs, les modalités d'approvisionnement des CTA, l'histoire de la production locale de médicaments au Ghana et les enjeux inhérents à la construction des politiques pharmaceutiques. Des observations directes ont été conduites à l'occasion de réunions entre les institutions nationales et les acteurs transnationaux ${ }^{5}$. Un travail au sein des archives nationales à Accra a permis de retracer l'histoire de la politique industrielle pharmaceutique du Ghana.

Nous allons tout d'abord analyser les logiques du Fonds mondial et de la PMI, ainsi que leurs stratégies pour la mise à disposition des CTA et leurs conséquences sur les systèmes pharmaceutiques des pays, puis nous nous attacherons à analyser les dispositifs institutionnels nationaux mis en œuvre par les États avec le soutien des acteurs transnationaux.

Logiques et stratégies des acteurs transnationaux : contrôle supranational et centralisation des achats

\section{Les logiques d'optimisation de performance des acteurs transnationaux}

Lors de sa création, le Fonds mondial se présente comme un " "mécanisme innovant", souple et léger, afin de se détacher des

agreement $\mathrm{n}^{\circ} 337372$. Pour plus de détails: http://globalmed.ird.fr/ (consulté le 25 mai 2018).

${ }^{4}$ Une approbation de recherche a été obtenue de la part des comités nationaux d'éthique du Bénin ( $n^{\circ} 30$ du 02/12/2013) et du Ghana ( $n^{\circ}$ GHS-ERC : 10/05/2014). Les enquêtés ont donné oralement leur consentement éclairé.

${ }^{5}$ Ces réunions incluent la révision annuelle du Malaria Operational Plan (MOP) de la PMI en mai 2015 à Accra, l'atelier en décembre 2014 au Bénin pour la rédaction de la note conceptuelle du Programme national de lutte contre le paludisme auprès du Fonds mondial dans le cadre du nouveau modèle de financement, et les $2^{\text {es }}$ assises du Comité national des approvisionnements en produits de santé du Bénin qui se sont tenues en octobre 2014 à Cotonou. 
organisations internationales et des critiques qui leurs sont adressées " (Eboko et al., 2015: 62). Il permet de mobiliser des fonds à l'échelle mondiale et de les mettre à disposition des États (Kerouedan, 2011). II s'inscrit dans le nouveau paradigme de l'aide publique au développement qui promeut l'alignement sur les besoins et les demandes des pays, et non l'inverse, dans le respect des principes de la déclaration de Paris (Eboko et al., 2015). Ainsi les demandes de financement auprès du Fonds mondial sont élaborées par des instances de coordination nationale qui regroupent un large éventail d'acteurs tels que des ONG, des représentants de la société civile, du secteur privé et des ministères. Faisant suite à la crise financière mondiale de 2008 et aux révélations de détournement de fonds dans certains pays (Eboko et al., 2015), le Fonds mondial traverse à partir de 2009 une grave crise économique qui le conduit à adopter en 2014 un nouveau modèle de financement (Fonds mondial, 2014). II s'accompagne de l'obligation pour les pays de recourir au mécanisme de groupement des achats souhaité par le Fonds mondial. Dans le cadre du nouveau modèle de financement, ce n'est désormais plus aux pays de définir le montant des subventions nécessaires à leurs actions, mais au Fonds mondial de leur attribuer des budgets calculés en fonction de critères comme la charge nationale de morbidité de la maladie, le niveau de revenu du pays et les financements externes dont il bénéficie (Fonds mondial, 2014). Le nouveau modèle de financement s'inscrit dans la stratégie du Fonds mondial d' "investir pour l'impact ", visant à améliorer la façon par laquelle I'institution évalue, approuve et contrôle les subventions. II répond à une logique d'optimisation des investissements publics et d'évaluation de la performance (Gaudillière, 2016), plutôt que de favoriser une plus grande autonomie des institutions nationales.

À partir de 2010, le secteur privé ghanéen est approvisionné en partie par des CTA subventionnées à travers l'Affordable Medicines Facilitymalaria $(\mathrm{AMFm})^{6}$. Ce programme pilote financé et mis en œuvre par le

\footnotetext{
${ }^{6}$ Tout comme six autres pays africains (le Kenya, Madagascar, le Niger, le Nigeria, l'Ouganda et la Tanzanie) et le Cambodge, le Ghana bénéficie entre 2010 et 2012 de la phase pilote de l'AMFm. Testé pendant deux ans, le programme sera reconduit en 2014 sous le nom de Private Sector Copayment Mechanism (PSCM) et ouvert à tous les pays bénéficiaires des subventions du Fonds mondial dans le cadre du nouveau mécanisme de financement.
} 
Fonds mondial a pour objectif de favoriser l'utilisation des CTA en influençant leur coût afin de les rendre autant accessibles que les anciens traitements. Pour ce faire, l'AMFm s'appuie sur les acteurs du marché privé (grossistes, détaillants) et privilégie une logique d'optimisation des investissements. Par un mécanisme de cofinancement, l'AMFm prend en charge près de $95 \%$ du prix des CTA auprès de six firmes pharmaceutiques multinationales (Ajanta Pharma, Cipla, Guilin, Ipca, Novartis et SanofiAventis) dont les CTA sont préqualifiées par l'OMS et auprès desquelles le Fonds mondial a négocié une baisse du prix de $80 \%$. Les CTA produites au Ghana et non préqualifiées par l'OMS sont exclues de ce dispositif. Les CTA de l'AMFm sont vendues à 1,5 cédi ghanéen $(\mathrm{GHC})$ - équivalent à 1 dollar par traitement sur le marché privé, alors que le prix des CTA produites localement par la firme Danadams par exemple, varie entre 3,4 GHC et $5,4 \mathrm{GHC}$, soit en moyenne deux à trois fois plus. En introduisant sur le marché privé, jusqu'alors réservé pour partie aux industriels ghanéens, des CTA préqualifiées par l'OMS et beaucoup moins chères, l'AMFm a contraint les firmes locales à interrompre leur production de CTA, comme en témoigne un industriel ghanéen :

"When we were about to start the production, we found that the Global Fund has come in the picture and AMFm program has been implemented under which $95 \%$ of the price is subsidized, so definitely we cannot survive, so we abandon the idea of manufacturing our product " (entretien réalisé le 22 avril 2015 à Accra).

Pour intervenir dans les pays, la PMI privilégie une logique d'externalisation de ses activités sous la forme de sous-traitance auprès d'ONG américaines sélectionnées par appel d'offres. À titre d'exemple au Bénin, PMI a financé pendant cinq ans à partir de 2011 le consortium ARM-3 (Accélération de la réduction de la morbidité et de la mortalité dues au paludisme/malaria), créé spécialement pour l'occasion par des ONG américaines et qui ont été sélectionnées à partir de critères de gestion et de performance. Chacune des ONG prend en charge les activités de son champ d'expertise ${ }^{7}$. L'externalisation et la contractualisation répondent à

${ }^{7}$ C'est ainsi que la composante de santé communautaire est assurée par l'ONG Africare, le renforcement de la prise en charge effectué par Medical Care Development International (qui 
une volonté d'assurer un contrôle sur les actions menées et les fonds mobilisés, dans une logique de performance des investissements. La PMI est financée par des subventions publiques que le Congrès doit justifier auprès des contribuables américains et qui nécessite le déploiement de dispositifs de contrôle et d'évaluation. Cette évaluation médicoéconomique mettant en rapport résultats et coûts, a pour but d'apprécier la performance des interventions et de garantir aux donateurs " d'en avoir pour leur argent » (Gaudillière, $2016: 124)$.

Les éléments présentés nous éclairent sur les contradictions soulevées par le nouveau modèle de financement du Fonds mondial, entre le discours officiel de la déclaration de Paris en faveur du renforcement institutionnel et les pratiques de contrôle supranational qu'il met en place. Ces nouvelles règles s'alignent sur les logiques de fonctionnement de PMI, issues des réformes de l'administration publique liées au New Public Management (NPM) et qui visent à pallier la supposée inefficacité des organisations publiques en appliquant des méthodes de gestion issues du secteur privé. Se retrouvent ainsi dans le fonctionnement de PMI et du Fonds mondial, les composantes du NPM telles que le " managérialisme » qui promeut une meilleure maîtrise des coûts, la " responsabilisation » qui favorise la création de structures autonomes, et la " contractualisation » qui encourage l'instauration de partenariats public-privé (Abord de Chatillon et Desmarais, 2012 : 11), tels qu'ARM-3 au Bénin. L'évaluation et la "culture de la performance " (Gaudillière, 2016:115) sont aussi très fortement ancrées dans les pratiques du Fonds mondial et de la PMI.

\section{Des mécanismes d'achat presque entièrement centralisés par les acteurs transnationaux}

Lors de l'adoption du nouveau modèle de financement, le Fonds mondial impose aux pays le recours à un mécanisme d'approvisionnement groupé, le Voluntary Pooled Procurement, qui permet des achats en très grandes quantités pour tous les pays et ainsi de réaliser des économies d'échelle. 
L'unité d'approvisionnement du ministère de la Santé du Ghana se voit alors retirer la responsabilité des achats de CTA qu'elle réalisait pourtant depuis 2008 au prétexte de son manque d'efficacité ${ }^{8}$. Au Bénin, le Fonds mondial consent à une exception pour l'achat des CTA, puisque l'essentiel de ses financements est consacré à approvisionner le pays en moustiquaires (Fonds mondial, 2015). Le budget alloué à deux ONG américaines, Catholic Relief Services (CRS) et Africare ${ }^{9}$, pour l'achat des CTA est marginal par rapport au budget global alloué au pays (1,7 million d'euros soit $7 \%$ du budget). Vu les faibles montants, les ONG sont autorisées à contractualiser avec la CAME pour la gestion du processus d'achat des CTA. La CAME est ainsi sous-traitante de tous les aspects administratifs et logistiques de la procédure d'achat, réalisée à travers un appel d'offres international. La sélection du vendeur revient aux deux ONG qui paient directement les fournisseurs sélectionnés; à aucun moment l'argent ne transite par la CAME, qui est rémunérée par CRS et Africare pour la gestion du processus d'achat, ce qui répond à une logique $\mathrm{d}^{\prime}$ externalisation ${ }^{10}$.

${ }^{8}$ Des délais dans les procédures auraient entraîné des retards de livraison des médicaments conduisant parfois à des ruptures de stock (information recueillie auprès du responsable logistique du Programme national de lutte contre le paludisme à Accra en avril 2015).

9 L'ONG Africare a été créée en 1970 aux États-Unis. Elle se prévaut de promouvoir la participation active des communautés et de créer des partenariats avec des organisations locales afin de contribuer au renforcement institutionnel des pays. Source: https:// www.africare.org/who-we-are/ (consulté le 25 mai 2018). Catholic Relief Services (CRS) est une ONG américaine de confession catholique créée en 1943. Elle intervient dans les domaines de l'agriculture et de la santé et se prévaut de favoriser la mobilisation des communautés dans ses modes d'action. Source: https://www.crs.org/ (consulté le 25 mai 2018). L'ONG Africare est soutenue financièrement à la fois par le Fonds mondial et par la $\mathrm{PMI}$, à travers sa participation au consortium ARM-3, soulignant "les récurrences structurelles de l'action transnationale en Afrique » (Eboko, $2015: 7$ ).

10 Informations recueillies à l'occasion d'entretiens conduits à Cotonou en octobre 2014 auprès du pharmacien en charge de la gestion des approvisionnements et des stocks respectivement pour CRS et Africare. Le processus a par ailleurs été décrit par l'assistant technique français en poste auprès de la CAME entre 2015 et 2016 pour le compte du Fonds mondial. Sa mission consistait au renforcement du système d'assurance qualité de la CAME et à l'harmonisation des dossiers d'appels d'offres. 
La PMI, pour sa part, confie l'achat des CTA à Deliver, un projet de renforcement de la chaîne d'approvisionnement financé par I'USAID ${ }^{11}$ et coordonné par John Snow, Inc., une société de consultance en santé publique basée aux États-Unis. Deliver approvisionne en CTA les 18 pays soutenus par la PMI, dont le Bénin et le Ghana. II présente des caractéristiques similaires au Voluntary Pooled Procurement du Fonds mondial, laissant en grande partie les États en marge du processus d'achat des médicaments. Les pays sont mobilisés seulement pour réceptionner les CTA qui sont stockées dans les structures d'entreposage de la CAME à Cotonou et du Central Medical Store à Accra. La distribution à travers les formations sanitaires publiques du pays est assurée par les programmes nationaux de lutte contre le paludisme, PNLP au Bénin et MNCP (Malaria National Control Program) au Ghana.

Le Fonds mondial et la PMI justifient leurs stratégies d'externalisation auprès de groupements d'achat afin de bénéficier de prix avantageux et de garantir la qualité des médicaments (Fonds mondial, 2010). II faut préciser que les achats de médicaments représentent des volumes financiers très importants au regard des budgets globaux. À titre d'exemple pour l'année 2014 au Bénin, le budget de la PMI pour l'achat de CTA s'élevait à plus de 2 millions de dollars, équivalant à $12,5 \%$ du budget total alloué au pays (PMI, 2014b). Entre 2008 et 2014, Deliver a conclu pour le compte du Bénin 16 contrats dont 14 avec la firme Novartis pour l'achat du Coartem ${ }^{\circledR 12}$ qu'elle produit en Chine et aux États-Unis (Spar et DeLacey, 2008). Au-delà de la logique de performance et de baisse des coûts affichée par les acteurs transnationaux, le contrôle sur les achats de médicaments répond donc aussi à des logiques commerciales et marchandes. La mainmise sur les achats de CTA permet au programme bilatéral des États-Unis de favoriser les multinationales basées sur son territoire, telles que Novartis, et offre plus largement la possibilité aux

\footnotetext{
${ }^{11}$ Informations recueillies lors de l'entretien mené le 22 octobre 2014 auprès du Commodities Logistics Specialist de I'USAID à Cotonou au Bénin et le 28 janvier 2015 à Accra au Ghana auprès de la personne en charge de la gestion des intrants de lutte contre le paludisme pour Deliver.

${ }^{12}$ Ces éléments sont tirés des contrats que nous nous sommes procurés auprès de la PMI au Bénin. Seulement deux contrats ont été confiés à la firme indienne Ipca en 2012.
} 
États de concilier l'aide au développement à la défense de leurs intérêts économiques (Ndour, 2013). En 2001, l'OMS signait un accord de 10 ans avec Novartis prévoyant que la firme cède à prix coûtant le Coartem ${ }^{\circledR}$ pour en approvisionner les secteurs publics ${ }^{13}$. Si l'accord permet à l'OMS de centraliser et de réaliser les achats de Coartem ${ }^{\circledR}$ pour le compte des pays et ainsi de bénéficier de prix plus avantageux (Cassier et Haxaire, 2014), il contribue aussi à la mainmise des acteurs transnationaux sur le contrôle des achats de CTA en offrant la possibilité pour les organisations internationales travaillant pour le compte des pays de bénéficier des mêmes conditions de la part de Novartis et de réaliser des achats.

C'est ainsi que, alors même que le Bénin et le Ghana disposent de systèmes nationaux d'approvisionnement de médicaments, le Fonds mondial et la PMI n'y recourent pas et privilégient leurs propres mécanismes d'achats groupés. De ce fait, ils génèrent des dispositifs parallèles à ceux des pays, entrant en concurrence directe avec la CAME au Bénin et l'unité d'approvisionnement du ministère de la Santé au Ghana. Ils contribuent à une multiplication des circuits d'achat qui se juxtaposent et conduisent à une fragmentation du système d'approvisionnement à l'origine, comme nous allons le voir, de difficultés dans la gestion de la mise à disposition des médicaments par les pays (Babaley, 2010). S'il ne fait nul doute que ces dispositifs permettent d'acheter les médicaments à plus bas prix grâce aux achats groupés, leur efficacité reste discutable. Les résultats d'une évaluation conduite par l'OMS en $2009^{14}$, confirmés par notre étude, montrent que la multiplication des circuits d'achat de médicaments générée par les acteurs transnationaux conduit à une fragmentation du système d'approvisionnement. II en découle des dysfonctionnements importants dans la gestion des médicaments au niveau

\footnotetext{
${ }^{13}$ Jusqu'en 2008, le Coartem ${ }^{\circledR}$ est la seule CTA produite sous la forme de combinaison à dose fixe (les deux molécules dans le même comprimé) et préqualifiée par l'OMS.

${ }_{14}$ L'OMS a réalisé en 2009 une évaluation des systèmes d'approvisionnement et de distribution des produits pharmaceutiques dans 13 pays (Ghana, Cameroun, Sénégal, Rwanda, Burundi, Mali, Congo-Brazzaville, Madagascar, RDC, Nigeria, Tanzanie, Tchad et Zambie). Alors qu'il existe des systèmes nationaux d'approvisionnement et de distribution des médicaments dans chaque pays, l'étude souligne l'existence en moyenne par pays, et pas seulement pour le paludisme, de 17 donateurs, 19 agences internationales d'approvisionnement et 84 circuits de distribution (Babaley, 2010).
} 
national tels que des ruptures de stock, des surstocks et des péremptions de produits (Babaley, 2010).

Le PNLP au Bénin et le NMCP au Ghana sont impliqués seulement à l'étape de la quantification permettant de définir les besoins annuels en CTA, d'identifier les manques de financements et de définir un plan d'approvisionnement. L'enjeu est important puisque les financements internationaux découlent de l'estimation des besoins. Si les programmes nationaux coordonnent l'exercice de quantification, assistés par le Fonds mondial et la PMI, ils ne disposent pas en revanche des éléments nécessaires à la gestion et à l'organisation de la mise à disposition des CTA, tels que les quantités commandées par les acteurs transnationaux, les délais d'approvisionnement et les dates de livraison prévues. Ils ne peuvent dès lors pas anticiper les ruptures de stock, voire les surstocks, ni même assurer une distribution appropriée des CTA dans le pays, ce qui relève pourtant de leur responsabilité. N'étant pas impliqués d'un bout à l'autre de la chaîne d'approvisionnement, les États n'ont alors qu'une très faible marge de manœuvre pour déployer des stratégies afin de pallier ces dysfonctionnements. Le Fonds mondial et la PMI cherchent néanmoins à impulser certaines formes d'appropriation au niveau des pays en soutenant la mise en place de dispositifs de renforcement des capacités nationales afin de répondre aux problèmes d'inefficacité des systèmes d'approvisionnement générés par leurs politiques d'achat et auxquels ils sont confrontés.

Les éléments empiriques présentés montrent que la multiplicité des acteurs transnationaux, l'importance de leurs moyens financiers et leur influence sur les politiques d'achat des CTA constituent autant d'obstacles à surmonter pour le Bénin et le Ghana dans l'appropriation de leurs politiques de mise à disposition des antipaludiques. La gouvernance de la santé globale produit des contraintes structurelles qui entravent le contrôle des États sur la sélection et la planification des priorités de leur politique de santé (Gautier et Ridde, 2017). Parmi les moyens à la portée des États pour la mise en application de la déclaration de Paris, Devi Sridhar (2009) propose le renforcement des mécanismes de responsabilisation des acteurs transnationaux et la création d'un espace politique permettant aux États d'élaborer des plans nationaux et de cultiver leur leadership national en matière de santé. Nous allons voir dans 
la partie suivante comment ces recommandations se déclinent localement au Bénin et au Ghana.

Des dispositifs institutionnels nationaux d'appropriation largement pilotés par les acteurs transnationaux

Des tentatives limitées au Bénin à la reprise du contrôle sur les importations de CTA

Au Bénin, deux mécanismes spécifiques ont été mis en place pour responsabiliser les acteurs transnationaux et s'assurer que les principes d'alignement, d'harmonisation et de cohérence soient respectés dans la mise à disposition des produits de santé. II s'agit du Comité national des approvisionnements en produits de santé (CNAPS) et du Panier commun. Ces deux mécanismes sont pour partie insufflés par les acteurs transnationaux qui jouent un rôle majeur dans leur élaboration et leur mise en œuvre par le soutien financier et technique qu'ils apportent. L'instauration du CNAPS découle d'une recommandation de l'OMS. Reconnu comme " bonne pratique " $^{15}$, le CNAPS a été créé en 2012 au Bénin par un arrêté ministériel faisant suite à une mission d'évaluation du système d'approvisionnement et de distribution des médicaments, réalisée en 2009 par un assistant technique et financée par le Fonds mondial. Elle pointait alors les conséquences délétères de la prolifération des programmes internationaux et de la fragmentation du système sur la capacité de l'État béninois à maîtriser et coordonner ses politiques (DPMED, 2014a). La DPMED, l'agence de régulation du Bénin, est alors désignée pour assurer la coordination du CNAPS. Ses employés reçoivent en 2014 le soutien d'un assistant technique français dont la mission d'une année, financée par le Fonds mondial, consiste à la mise en place d'une cellule chargée de l'animation du CNAPS qui, bien qu'ayant été créé deux ans plus tôt, n'était toujours pas fonctionnel. Le Panier commun a été mis en place par le PNLP avec le soutien de la PMI faisant suite à l'évaluation de la chaîne

${ }^{15}$ Recommandé par l'OMS à l'origine dans le cadre de l'épidémie du VIH-sida pour tous les pays, le CNAPS a été étendu à l'ensemble des programmes nationaux comme celui de la lutte contre le paludisme ou la tuberculose. 
d'approvisionnement des médicaments antipaludiques conduite par le programme bilatéral des États-Unis en 2012. La PMI a largement contribué techniquement et financièrement à la rédaction des "Procédures opérationnelles standardisées de gestion des intrants de lutte contre le paludisme suivant le mécanisme du "panier commun" " (PNLP, 2015) et à leur mise en œuvre. Une description détaillée de ces dispositifs institutionnels est nécessaire pour comprendre comment ces dispositifs locaux, bien que soutenus par les acteurs transnationaux, participent dans une certaine mesure à la formulation de la politique pharmaceutique nationale.

Le CNAPS est un dispositif permettant de centraliser les informations nécessaires à l'organisation de la mise à disposition des produits de santé. La DPMED en assure la coordination générale. Des sous-comités techniques sont créés, dont l'un dédié au paludisme et à la gestion des CTA est coordonné par le PNLP. Son rôle consiste à suivre la disponibilité des CTA et à alerter en cas de ruptures ou de surstocks (DPMED, 2014b). À travers sa mission de pilotage du CNAPS, la DPMED développe des documents normatifs visant à définir les rôles et les responsabilités des différents acteurs, à encadrer leurs pratiques d'approvisionnement et de distribution et à limiter les effets de fragmentation (DPMED, 2014b).

Le CNAPS, recommandé par l'OMS et mis en œuvre avec le soutien du Fonds mondial, constitue un dispositif de renforcement des pouvoirs nationaux face aux acteurs transnationaux et permet le développement d'un leadership national. II s'inscrit dans la création d'un espace politique permettant à l'État d'élaborer ses plans nationaux, sur lesquels les programmes de santé globale doivent s'aligner (Sridhar, 2009).

Pour pallier les ruptures de stock et les surstocks en CTA, conséquences du manque de coordination entre les acteurs lors de leur mise à disposition des CTA, le PNLP a adopté en août 2015 « Les procédures opérationnelles standardisées de gestion des intrants de lutte contre le paludisme suivant le mécanisme du "panier commun" »(PNLP, 2015) élaborées avec le soutien de la PMI. Ces procédures ne constituent pas à proprement parler un "panier commun", au sens où tous les programmes internationaux contribueraient financièrement auprès du pays afin que ce dernier achète les médicaments. Dans ce cas-ci, il permet au PNLP la mise en commun des différents stocks de CTA achetées par les acteurs transnationaux et 
stockées à la CAME. Ce stock du Panier commun dénie alors tout droit de propriété sur les produits, et les CTA sont gérées indistinctement quel que soit le partenaire les ayant achetées (PNLP, 2015). Il permet au PNLP de distribuer les médicaments en fonction des besoins et est censé ainsi éviter les ruptures de stock, dues en partie au manque de coordination entre les acteurs transnationaux lors des achats et des livraisons de CTA. Les acteurs transnationaux sont tenus d'informer le PNLP et la CAME de l'expédition des médicaments et de leur fournir les documents ainsi que la date prévisionnelle de livraison. Ils doivent aussi informer le PNLP tous les mois du statut des commandes et anticiper les retards.

Les procédures du Panier commun constituent en théorie là aussi un moyen pour l'État béninois de regagner un certain degré de maîtrise sur ses politiques de mise à disposition des CTA en lui donnant le pouvoir de faire pression sur les acteurs transnationaux, afin que ceux-ci respectent leurs engagements en termes de quantités et d'échéances de livraison des CTA. La mise en place du Panier commun, largement soutenu par la PMI, contribue en ce sens à un changement d'équilibre des pouvoirs entre acteurs, tout en soulignant le besoin de responsabilité mutuelle (Esser, 2014). Il pourrait permettre une relocalisation du suivi et du contrôle de la mise à disposition des CTA auprès du PNLP. Or, s'il est encore trop tôt pour dresser un bilan de ces deux dispositifs, dans les faits nos observations démontrent qu'ils n'ont jusqu'à présent pas rempli les objectifs affichés. Par exemple, en 2016 la Banque mondiale n'a pas respecté les procédures du Panier commun en réalisant un achat de CTA non planifié et non concerté, représentant un volume de médicaments bien supérieur aux besoins réels et aux dates de péremption très courtes ${ }^{16}$. En conséquence, le PNLP, la CAME et la PMI ont été dans l'obligation de mettre en place un plan d'urgence afin d'écouler les surstocks de CTA, en proposant de les distribuer dans les pharmacies privées. Si en théorie ces dispositifs institutionnels devraient permettre de renforcer les capacités nationales du Bénin pour la mise à disposition des CTA, cette situation démontre que dans les faits leur efficacité est discutable.

\footnotetext{
${ }^{16}$ Nos informateurs nous ont précisé en août 2016 que les CTA avaient été livrées dans le pays en avril de cette même année avec des dates de péremption d'octobre-novembre 2016 et de janvier 2017.
} 
Comme nous l'avons montré, les dispositifs institutionnels déployés au Bénin par la DPMED et le PNLP sont en grande partie insufflés par la PMI et le Fonds mondial qui en contrôlent largement aussi l'élaboration technique et financière. Mais, alors que l'implication du PNLP d'un bout à l'autre de la chaîne d'approvisionnement semble essentielle à la maîtrise des données nécessaires à l'organisation de la mise à disposition des CTA, les dispositifs déployés portent principalement sur le stockage et la redistribution des CTA et finalement très peu sur les politiques d'achat et de choix des fournisseurs qui, en raison des intérêts financiers en jeu, restent largement contrôlées par la PMI et le Fonds mondial, comme en témoigne une pharmacienne du projet ARM-3 :

"C'est une volonté de I'USAID-PMI qui ne veut pas donner son volet d'achat à qui que ce soit dans tous les pays où ils interviennent » (entretien réalisé le 3 octobre 2014 à Cotonou).

Dès lors, on observe que la mise en œuvre de l'appropriation est ainsi traversée de nombreuses contradictions découlant des relations de pouvoir entre les acteurs (Raffinot, 2010). De plus, l'absence de clarté conceptuelle (Esser, 2014), le flou sémantique et "les fondements théoriques et empiriques peu explicités " (Raffinot, 2010 : 90) du concept d'appropriation conduisent à la définition et à la mise en œuvre de stratégies d'appropriation au sein desquelles les acteurs transnationaux continuent de jouer un rôle majeur, et qui sont qualifiées de "donordriven ownership » (Bergamaschi, 2008).

Les éléments empiriques présentés démontrent que les dispositifs déployés au Bénin contribuent dans une moindre mesure au renforcement des pouvoirs nationaux en permettant de favoriser une intégration locale, bien que limitée, des systèmes d'approvisionnement par le PNLP, la CAME et la DPMED. Ils constituent aussi des dispositifs locaux de formulation des politiques pour la mise à disposition des CTA. Au Ghana, le gouvernement déploie avec le soutien des acteurs transnationaux d'autres types de dispositifs et ce sont principalement les initiatives de l'État ghanéen autour du développement d'une production locale de CTA que nous allons analyser. 


\section{Les défis du développement autonome d'une production locale de médicaments au Ghana}

Les effets néfastes de l'AMFm sur la production locale de CTA au Ghana ont conduit l'agence ghanéenne de régulation, la FDA-Ghana, à développer à partir de 2013 une feuille de route (la Road map) afin d'accompagner les firmes locales dans le processus de mise aux normes des Bonnes Pratiques de Fabrication (BPF) de l'OMS (WHO, 2007) d'ici la fin de l'année 2020. Si l'objectif principal de la FDA-Ghana à travers ce programme est de s'assurer de la qualité des médicaments produits, elle vise aussi à accompagner les firmes ghanéennes dans leur quête de la préqualification de l'OMS pour l'accès aux marchés des médicaments génériques subventionnés. En effet, l'enquête conduite sur le terrain montre qu'aucun des sites de production de médicaments au Ghana n'est conforme aux normes internationales des BPF comme cela est exigé par l'OMS dans le processus de préqualification.

Bien que la FDA-Ghana soit à l'origine de la Road map, elle bénéficie du soutien de divers acteurs transnationaux. L'Organisation des Nations unies pour le développement industriel (ONUDI) s'est associée à la FDA-Ghana à partir de 2015, en proposant son expertise technique et son expérience d'un projet similaire au Kenya. La FDA-Ghana bénéficie de financements du département britannique du Développement international (DFID) pour les formations qu'elle organise auprès des industries locales. La United States Pharmacopeial Convention (USP), une organisation qui vise à améliorer la qualité des médicaments, assiste la FDA-Ghana dans l'élaboration des ateliers et prend en charge la formation de ses inspecteurs sur les BPF. L'OMS apporte une expertise technique. L'agence allemande de coopération (GIZ) finance un projet dont l'objectif est d'encourager la localisation au Ghana de la production de firmes multinationales. La firme LaGray a ainsi bénéficié de ce partenariat qui lui permet un accès à la technologie et à du personnel compétent. La GIZ a aussi financé une étude de faisabilité pour la création au Ghana d'un centre régional d'études de bioéquivalence, afin de permettre aux firmes ghanéennes de faire réaliser ces études localement et à moindre coût. La preuve de la bioéquivalence est nécessaire à la préqualification par l'OMS de médicaments génériques. 
Le soutien à la production locale de médicaments au Ghana s'inscrit dans le cadre des politiques de développement portées par les agences d'aide bilatérale de certains États européens tels que l'Angleterre et l'Allemagne, qui privilégient les modèles favorisant le soutien direct aux États ainsi que le renforcement des systèmes nationaux de santé (Storeng, 2014). Les firmes ghanéennes désireuses d'obtenir la préqualification OMS collaborent très sérieusement à la Road map, en tentant d'investir dans la construction de nouvelles usines (UNIDO, 2015). Mais les processus de normalisation industrielle sont longs et demandent des investissements importants. Or l'accès au capital nécessaire demeure un frein pour les firmes ghanéennes et aucun des acteurs transnationaux ne prévoit de financement en la matière.

Les tentatives de développement d'une politique pharmaceutique industrielle par le Ghana sont sous l'influence de circulations transnationales humaines et technologiques de la GIZ, de I'ONUDI, de I'OMS, du DFID et de I'USP. Dans leur démarche de renforcement des capacités locales, ces acteurs transnationaux se heurtent là aussi aux ambivalences des politiques de l'aide internationale. Les critères de gestion et de contrôle, la culture de la performance et les pratiques d'achats groupés à l'étranger pour profiter des prix les plus bas, laissent en effet peu d'espace aux États pour l'achat de médicaments antipaludiques produits localement. Ainsi, la politique industrielle du Ghana est elle aussi affectée par les contradictions inhérentes aux politiques d'appropriation : alors que certains acteurs transnationaux, tels que le Fonds mondial et la PMI, recourent à des dispositifs d'achats externalisés auprès de multinationales pour la mise à disposition de CTA, d'autres acteurs transnationaux assistent les entreprises locales dans leur mise aux normes.

\section{Conclusion}

Le Bénin et le Ghana déploient tous deux, avec le soutien des acteurs transnationaux, des dispositifs institutionnels qui leur permettent en théorie de maîtriser les politiques de mise à disposition des CTA. Or, les critères de gestion et d'évaluation du Fonds mondial et de la PMI ainsi que leurs politiques d'achat de médicaments, limitent dans les faits l'autonomie octroyée aux pays. Ce paradoxe, relevé aussi bien au Bénin 
qu'au Ghana, découle de la contradiction structurelle de l'aide internationale qui oscille entre le principe d'une plus grande autonomie des institutions nationales, prônée par l'objectif de l'appropriation, et celui d'une plus grande efficacité de sa mise en œuvre, en réponse aux logiques internes des institutions internationales.

Dans les deux cas, et ce indistinctement du niveau de développement économique et industriel des pays, les États du Bénin et du Ghana ont finalement assez peu d'emprise sur les dispositifs déployés dont ils ne contrôlent que partiellement la définition et la mise en œuvre largement dominées par les acteurs transnationaux. Ils demeurent à une position subalterne dans le processus de construction de leurs propres politiques, ce qui s'explique par l'asymétrie de moyens financiers et de pouvoir entre les États et les acteurs transnationaux, ainsi que par le mode de gouvernance centralisé de la santé globale. Comme l'a démontré Bergamaschi (2008), les réformes engagées suite à la déclaration de Paris pour modifier les rapports de force entre les différents acteurs sont largement insuffisantes et contribuent même à donner aux acteurs transnationaux plus de pouvoir dans le processus de prise de décision, notamment par le rôle qu'ils exercent dans la définition des politiques d'appropriation. La réelle mise en application du concept d'appropriation conduirait en effet à un changement dans les relations de pouvoir qui sont aujourd'hui largement en faveur des acteurs transnationaux. Ces derniers semblent ainsi peu disposés à respecter pleinement leurs engagements et à laisser le temps (Bergamaschi, 2008) et l'espace (Sridhar, 2009) nécessaires à l'émergence de l'appropriation des politiques par les pays, comme dans le cas du Ghana où la construction d'un tissu industriel pharmaceutique aux normes internationales nécessite du temps et des investissements auxquels les acteurs transnationaux ne sont pas prêts à consentir.

L'émergence des dispositifs institutionnels au Bénin et au Ghana met ainsi en exergue l'usage stratégique et les limites fondamentales des politiques d'appropriation dès lors que les institutions internationales en sont juge et partie (Raffinot, 2010). Toutefois, cela ne signifie pas pour autant l'absence totale de constructions locales de politiques pour reprendre le contrôle sur la mise à disposition des CTA. Le CNAPS et le Panier commun au Bénin, la Road map au Ghana sont autant d'outils et de 
moyens à la disposition des gouvernements pour la formulation de leurs politiques dans des contextes nationaux très différents. Ainsi, l'enjeu de la réappropriation des pouvoirs de définition des programmes d'achat de médicaments s'inscrit plus largement dans la problématique du contrôle par les États de la définition de leur politique pharmaceutique nationale.

\section{Bibliographie}

ABORD DE CHATILLON E. et DESMARAIS C., 2012, "Le Nouveau Management Public est-il pathogène ? ", Management international, $16: 10-24$.

BABALEY M., 2010, "Les défis dans les systèmes d'approvisionnement et de distribution des médicaments ", Genève, OMS, 23 p.

BAXERRES C., 2013, Du médicament informel au médicament libéralisé: Une anthropologie du médicament pharmaceutique au Bénin, Paris, Les Éditions des archives contemporaines.

BERGAMASCHI I., 2008, Mali: patterns and Limits of Donor-driven ownership, Global Economic Governance Programme, working paper $n^{\circ} 41$, Oxford, Oxford University, $34 \mathrm{p}$.

CASSIER M. et CORREA M., 2009, "Éloge de la copie : le reverse engineering des antirétroviraux contre le $\mathrm{VIH} /$ sida dans les laboratoires pharmaceutiques brésiliens ", Sciences Sociales et Santé, 27(3) : 77-103.

CASSIER M. et HAXAIRE C., (septembre) 2014, " Histoire des antipaludiques, des CTA et des molécules associées ", Bénin, Globalmed, multigr.

CHAUDHURI S., 2015, "Can Foreign Firms Promote Local Production of Pharmaceuticals in Africa? ", in MACKINTOSH M., BANDA G., TIBANDEBAGE P. et WAMAE W. (éds), Making Medicines in Africa, Palgrave Macmillan : 103-121.

DPMED, (19-20 juin) 2014a, "Première session 2014 du Comité national des approvisionnements en produits de santé (CNAPS), rapport général ", Porto Novo, ministère de la Santé/DPMED, $26 \mathrm{p}$.

DPMED, (29 et 30 octobre) 2014b, « Synthèse deuxième session du Comité national des approvisionnements en produits de santé (CNAPS)", Cotonou, ministère de la Santé/DPMED, $3 \mathrm{p}$. 
EBOKO F., BOURDIER F. et BROQUA C., 2011, "Introduction ", in EBOKO F., BOURDIER F. et BROQUA C. (éds), Les Suds face au sida. Quand la société civile se mobilise, Marseille, IRD Éditions : 21-43.

EBOKO F., 2015, "Vers une matrice de l'action publique en Afrique ? Approche trans-sectorielle de l'action publique en Afrique contemporaine", Questions de Recherche, 45 : 1-40.

EBOKO F., HANE F., DEMANGE E. et FAYE S., 2015, "Gouvernance et sida en Afrique : instruments de l'action publique internationale, l'exemple du Fonds mondial ", Mondes en développement, 2(170) : 59-74.

ESSER D., 2014, "Elusive accountability in the VIH scale-up: "ownership" as a functional tautology ", Global Public Health, 9(1-2) : 43-56.

FONDS MONDIAL, 2010, "Initiatives Globales pour l'Accès aux médicaments ", Genève, TBS Meeting, Fonds mondial, $31 \mathrm{p}$.

FONDS MONDIAL, 2014, "Nouveau modèle de financement ", Genève, Fonds mondial, $200 \mathrm{p}$.

FONDS MONDIAL, 2015, « Concept note for Malaria-Benin », Bénin, Fonds mondial, $45 \mathrm{p}$.

GAUDILLIÈRE J.-P., 2016, « Un nouvel ordre sanitaire international ? Performance, néolibéralisme et outils du gouvernement médico-économique", Écologie \& politique, 1(52) : 107-124.

GAUTIER L. et RIDDE V., 2017, "Health financing policies in Sub-Saharan Africa: government ownership or donors' influence? A scoping review of policymaking processes ", Global Health Research and Policy, 2 : 23.

KEROUEDAN D., 2011, "Évolutions de l'architecture internationale de l'aide en faveur de la santé dans les pays en développement ", in KEROUEDAN D. (éd.), Santé Internationale, Paris, Presses de Sciences Po : 357-376.

LANTENOIS C. et CORIAT B., 2014, "La "préqualification" OMS: origines, déploiement et impacts sur la disponibilité des antirétroviraux dans les pays du Sud ", Sciences Sociales et Santé, 32 : 71-99.

MAHAMÉ S. et BAXERRES C., 2015, "Distribution grossiste du médicament en Afrique: fonctionnement, commerce et automédication. Regards croisés Bénin-Ghana ", in "Les actes des rencontres Nord/Sud de l'automédication et de ses déterminants ", https://hal.archivesouvertes.fr/hal-01336870/document (consulté le 25 mai 2018). 
NAUDET J.-D., 2000, "Le dilemme entre solidarité et dépendance ", Autrepart, 13 : 173-193.

NDOUR M., 2013, " La politisation de l'accès aux médicaments dans l'espace international. L'Organisation mondiale de la santé comme foyer d'une dynamique contestataire ", thèse de doctorat de science politique sous la direction de Johanna Siméant, université Paris 1 Panthéon Sorbonne, Paris, $562 \mathrm{p}$.

PNLP, (août) 2015, « Procédures opérationnelles standardisées de gestion des intrants de lutte contre le paludisme suivant le mécanisme du "Panier Commun" ", première édition, Cotonou, PNLP-USAID, 18 p.

PMI, 2014a, "Annual Performance Report. Fiscal year 2014, Program Year 3 », Cotonou, PMI, $48 \mathrm{p}$.

PMI, 2014b, « Malaria Operationnal Plan », Bénin, PMI, 78 p.

RAFFINOT M., 2010, «L'appropriation (ownership) des politiques de développement: de la théorie à la pratique ", Mondes en développement, $1(149): 87-104$.

SPAR D.L. et DELACEY B., 2008, "The Coartem Challenge », Harvard Business School, 9-706-037, $15 \mathrm{p}$.

SRIDHAR D., 2009, "Post-Accra: is there space for country ownership in global health? ", Third World Quarterly, 30(7) : 1363-1377.

STORENG T.K., 2014, "The GAVI Alliance and the 'Gates approach' to health system strengthening ", Global Public Health, 9(8) : 865-879.

UNIDO, 2015, "Ghana GMP Roadmap, A Stepwise Approach for the Pharmaceutical Industry to Attain WHO GMP Standards ", Draft, Accra.

WHO, 2007, «Quality assurance of pharmaceuticals. A compendium of guidelines and related materials, Volume 2, $2^{\text {nd }}$ updated edition. Good manufacturing practices and inspection ", Geneva, World Health Organization, $418 \mathrm{p}$. 


\section{Aiviviv}

Jessica Pourraz est docteure en santé et sciences sociales

MERIT, CERMES3, EHESS (France)

E-mail : jessica.pourraz@ird.fr

Carine Baxerres est chargée de recherche en anthropologie

MERIT, IRD, université Paris 5, Sorbonne Paris Cité et CNE, Marseille (France)

E-mail : carine.baxerres@ird.fr

Maurice Cassier est directeur de recherche au CNRS en sociologie

CERMES3 (France)

E-mail : maurice.cassier@cnrs.fr 